\title{
Weak $\theta$-contractions and some fixed point results with applications to fractal theory
}

\author{
Mohammad Imdad', Waleed M. Alfaqih ${ }^{1,2^{*}}$ (D) and Idrees A. Khan ${ }^{3}$
}

\section{"Correspondence:}

waleedmohd2016@gmail.com

'Department of Mathematics,

Aligarh Muslim University, Aligarh,

India

${ }^{2}$ Department of Mathematics,

Hajjah University, Hajjah, Yemen

Full list of author information is

available at the end of the article
The Author(s) 2018. This article is distributed under the terms of the Creative Commons Attribution 4.0 International License (http://creativecommons.org/licenses/by/4.0/), which permits unrestricted use, distribution, and reproduction in any medium, provided you give appropriate credit to the original author(s) and the source, provide a link to the Creative Commons license, and indicate if changes were made.

\section{Introduction}

The Banach contraction principle is one of the pivotal results of nonlinear analysis, which asserts that every contraction mapping defined on a complete metric space $(M, d)$ to itself admits a unique fixed point. This principle is a very effective and popular tool for guaranteeing the existence and uniqueness of solutions of certain problems arising within and beyond mathematics. The Banach contraction principle has been extended and generalized in many directions (see [1-6] and references therein). With a similar quest, beginning from a function $\theta:(0, \infty) \rightarrow(1, \infty)$ satisfying suitable properties (see Definition 3.1 to be given later), Jleli and Samet [5] proposed a new type of contractive mappings known as $\theta$-contraction (or $J S$-contraction) and proved a fixed point result in generalized metric spaces wherein the authors showed that the Banach contraction principle remains a particular case of $\theta$-contraction.

In this paper, we observe that the first condition in Definition 3.1 is unnecessarily stringent; its omission enlarges the class of functions $\theta:(0, \infty) \rightarrow(1, \infty)$. In fact, we consider the families $\Theta_{2,3}$ and $\Theta_{2,4}$ and utilize the same to define a weak $\theta$-contraction (see Definition 3.2 to be introduced shortly) such that every weak $\theta$-contraction on a complete metric space is a Picard operator. Also, we provide an example of a weak $\theta$-contraction that is not a Banach contraction.

The basic concept of fractal theory is the iterated function system (IFS) introduced by Hutchinson [7] and generalized by Barnsley [8], IFS being the main generator of fractals. This consists of a finite set of contractions $\left\{f_{i}\right\}_{i=1}^{N}$ on a complete metric space $(M, d)$ into itself. For such an IFS, there is always a unique nonempty compact set $A \subset M$ such that $A=\bigcup_{i=1}^{N} f_{i}(A)$, wherein generally $A$ is a fractal set called the attractor of the respective IFS. 
In Sect. 4, we apply Theorem 3.2 to obtain the existence and uniqueness of the attractor of some iterated function system on a complete metric space and, also, to provide an example to demonstrate our results.

The fixed point theory on metric spaces endowed with a binary relation is a relatively new area initiated by Turinici [9]. This area becomes very active after the appearance of the very interesting results of Ran and Reurings [1] and Nieto and Rodriguez-Lopez [2, 10] with their nice applications. Recently, this branch of fixed point theory has been developed by many researchers. To mention a few, we recall Bhaskar and Lakshmikantham [11], BenEl-Mechaiekh [12], Samet and Turinici [13], Alam and Imdad [14], Imdad et al. [15-17], and several others.

In Sect. 5, we provide some fixed point results on a generalized metric space equipped with a binary relation under weak $\theta$-contractions without completeness requirement. Also, we adopt some examples to exhibit the utility of our results.

Finally, in Sect. 6, we apply Theorems 5.2 and 5.3 to obtain the existence and uniqueness of an attractor for a countable iterated function system, which is also composed by contractions on a complete metric space besides furnishing an example to exhibit the validity of our results.

\section{Preliminaries}

In this section, we recall some notions, notations, and basic results.

Throughout this presentation, $\mathbb{N}$ is the set of natural numbers, and $\mathbb{N}_{0}=\mathbb{N} \cup\{0\}$. We write $\left\{u_{n}\right\} \rightarrow u$ whenever $\left\{u_{n}\right\}$ converges to $u$. If $M$ is a nonempty set, $u \in M$, and $f: M \rightarrow$ $M$, then we write $f u$ instead of $f(u)$. The sequence $\left\{u_{n}\right\}$ defined by $u_{n}=f^{n} u_{0}$ is called a Picard sequence based at the point $u_{0} \in M$.

Definition 2.1 ([18]) A self-mapping $f$ on a metric space $(M, d)$ is said to be $a$ Picard operator if it has a unique fixed point $z \in M$ and $z=\lim _{n \rightarrow \infty} f^{n} u$ for all $u \in M$.

Lemma 2.1 ([19]) Let $\left\{u_{n}\right\}$ be a sequence in a metric space $(M, d)$. If $\left\{u_{n}\right\}$ is not a Cauchy sequence, then there exist $\epsilon>0$ and two subsequences $\left\{u_{n(k)}\right\}$ and $\left\{u_{m(k)}\right\}$ of $\left\{u_{n}\right\}$ such that $k \leq m(k)<n(k), d\left(u_{m(k)}, u_{n(k)}\right) \geq \epsilon$ and $d\left(u_{m(k)}, u_{n(k)-1}\right)<\epsilon \forall k \in \mathbb{N}$.

Furthermore, $\lim _{k \rightarrow \infty} d\left(u_{m(k)}, u_{n(k)}\right)=\epsilon$, provided that $\lim _{n \rightarrow \infty} d\left(u_{n}, u_{n+1}\right)=0$.

Definition 2.2 Let $(M, d)$ be a metric space, and let $\mathcal{K}(M)$ the class of all nonempty compact subsets of $M$. The function $\eta: \mathcal{K}(M) \times \mathcal{K}(M) \rightarrow[0, \infty)$ defined by $\eta(A, B)=$ $\max \{D(A, B), D(B, A)\}$, where $D(A, B)=\sup _{u \in A} \inf _{v \in B} d(u, v)$, for all $A, B \in \mathcal{K}(M)$, is a metric known as Hausdorff-Pompeiu metric. It is well known that if $(M, d)$ is complete, then $(\mathcal{K}(M), \eta)$ is also complete.

Lemma 2.2 ([20]) Let $A, B, C \in \mathcal{K}(M)$. Then we have the following:

(i) $A \subset B$ if and only if $D(A, B)=0$;

(ii) $D(A, B) \leq D(A, C)+D(C, B)$.

Lemma 2.3 ([21]) If $\left\{E_{i}\right\}_{i \in \mathfrak{\Im}}$ and $\left\{F_{i}\right\}_{i \in \Im}$ are finite collections of elements in $\mathcal{K}(M)$, then

$$
\eta\left(\bigcup_{i \in \Im} E_{i}, \bigcup_{i \in \Im} F_{i}\right) \leq \sup _{i \in \Im} \eta\left(E_{i}, F_{i}\right)
$$


Definition 2.3 ([22]) Let $M$ be a nonempty set. A function $d: M \times M \rightarrow[0, \infty]$ is called a generalized metric on $M$ if it satisfies the following conditions (for all $u, v, z \in M$ ):

(G1) $d(u, v)=0$ if and only if $u=v$;

(G2) $d(u, v)=d(v, u)$;

(G3) $d(u, v) \leq d(u, z)+d(z, v)$.

The pair $(M, d)$ is called a generalized metric space. Moreover, if every Cauchy sequence in $M$ converges to a point in $M$, then $(M, d)$ is called a generalized complete metric space.

Wardowski [3] introduced a new class of auxiliary functions and utilized the same to define $F$-contractions as follows:

Definition 2.4 ([3]) Let $\mathcal{F}$ be the family of all functions $F:(0, \infty) \rightarrow \mathbb{R}$ satisfying the following conditions:

$F 1: F$ is strictly increasing;

$F 2$ : for every sequence $\left\{\alpha_{n}\right\} \subset(0, \infty)$,

$$
\lim _{n \rightarrow \infty} F\left(\alpha_{n}\right)=-\infty \Leftrightarrow \lim _{n \rightarrow \infty} \beta_{n}=0
$$

F3: there exists $k \in(0,1)$ such that $\lim _{\alpha \rightarrow 0^{+}} \alpha^{k} F(\alpha)=0$.

A self-mapping $f$ on a metric space $(M, d)$ is said to be an F-contraction if there exist $\tau>0$ and $F \in \mathcal{F}$ such that (for all $u, v \in M$ )

$$
d(f u, f v)>0 \quad \Rightarrow \quad \tau+F(d(f u, f v)) \leq F(d(u, v)) .
$$

Wardowski [3] proved that every $F$-contraction mapping on a complete metric space is a Picard operator. Thereafter, Piri and Kumam [23] replaced condition F3 by

$F 4: F$ is a continuous mapping.

\section{Weak $\theta$-contractions}

Definition 3.1 (see $[5,24]$ ) Let $\theta:(0, \infty) \rightarrow(1, \infty)$ be a function and consider the following conditions:

$\Theta 1: \theta$ is nondecreasing;

$\Theta 2$ : for each sequence $\left\{\alpha_{n}\right\}$ in $(0, \infty)$,

$$
\lim _{n \rightarrow \infty} \theta\left(\alpha_{n}\right)=1 \quad \Leftrightarrow \quad \lim _{n \rightarrow \infty} \alpha_{n}=0^{+}
$$

$\Theta 3$ : there exist $r \in(0,1)$ and $l \in(0, \infty]$ such that $\lim _{\alpha \rightarrow 0^{+}} \frac{\theta(\alpha)-1}{\alpha^{r}}=l$;

$\Theta 4: \theta$ is continuous.

We adopt the following notations in the sequel:

- $\Theta_{1,2,3}$, the family of all functions $\theta$ that satisfy $\Theta 1-\Theta 3$;

- $\Theta_{1,2,4}$, the family of all functions $\theta$ that satisfy $\Theta 1, \Theta 2$, and $\Theta 4$;

- $\Theta_{2,3}$, the family of all functions $\theta$ that satisfy $\Theta 2$ and $\Theta 3$;

- $\Theta_{2,4}$, the family of all functions $\theta$ that satisfy $\Theta 2$ and $\Theta 4$;

- $\Theta_{2}$, the family of all functions $\theta$ that satisfy $\Theta 2$.

Remark 3.1 In the following, we observe some relations between Definitions 2.4 and 3.1: 
(i) $\theta:(0, \infty) \rightarrow(1, \infty)$ satisfies, respectively, $\Theta 1, \Theta 2$, or $\Theta 4$ if and only if $\ln \ln \theta:(0, \infty) \rightarrow(-\infty, \infty)$ satisfies $F 1, F 2$, or $F 4$;

(ii) $F:(0, \infty) \rightarrow(-\infty, \infty)$ satisfies, respectively, $F 1, F 2$, or $F 4$ if and only if $e^{e^{F}}:(0, \infty) \rightarrow(1, \infty)$ satisfies $\Theta 1, \Theta 2$, or $\Theta 4$.

Example $3.1([5])$ Define $\theta:(0, \infty) \rightarrow(1, \infty)$ by $\theta(\alpha)=e^{\sqrt{\alpha}}$. Then $\theta \in \Theta_{1,2,3,4}$.

Example $3.2([5])$ Define $\theta:(0, \infty) \rightarrow(1, \infty)$ by $\theta(\alpha)=2-\frac{2}{\pi} \arctan \left(\frac{1}{\alpha^{r}}\right), 0<r<1$. Then $\theta \in \Theta_{1,2,3,4}$.

Example $3.3([24])$ Define $\theta:(0, \infty) \rightarrow(1, \infty)$ by $\theta(\alpha)=e^{\alpha}$. Then $\theta \in \Theta_{1,2,4}$.

Now, we add some more examples to this effect.

Example 3.4 Define $\theta:(0, \infty) \rightarrow(1, \infty)$ by $\theta(\alpha)=e^{\alpha e^{\frac{-1}{\alpha}}}$. Then $\theta \in \Theta_{1,2,4}$.

Example 3.5 Define $\theta:(0, \infty) \rightarrow(1, \infty)$ by $\theta(\alpha)=e^{\sqrt{\frac{\alpha}{2}+\sin \alpha}}$. Then $\theta \in \Theta_{2,3}$.

Example 3.6 The following functions $\theta:(0, \infty) \rightarrow(1, \infty)$ are in $\Theta_{2,4}$ :

$1 \theta(\alpha)=e^{\frac{\alpha}{2}+\sin \alpha}$;

$2 \theta(\alpha)=\alpha^{r}+1, r \in(0, \infty)$.

For more examples, see [5, 24].

Jleli and Samet [5] proved the following theorem.

Theorem 3.1 (see [5]) Let $(M, d)$ be a complete metric space, and let $f: M \rightarrow M$. Assume that there exist $\theta \in \Theta_{1,2,3}$ and $h \in(0,1)$ such that (for all $\left.u, v \in M\right)$

$$
d(f u, f v)>0 \Rightarrow \theta(d(f u, f v)) \leq[\theta(d(u, v))]^{h}
$$

Then $f$ has a unique fixed point.

The following proposition shows that $f$ in Theorem 3.1 is continuous due to $\Theta 2$.

Proposition 3.1 Let $(M, d)$ be a metric space, and let $f: M \rightarrow M$. If $f$ satisfies (3.1) for some $\theta \in \Theta_{2}$ and $h \in(0,1)$, then $f$ is continuous.

Proof Let $u, v \in M$ be such that $d(u, v) \rightarrow 0$. Then we must have $\theta(d(u, v)) \rightarrow 1$ (due to $\Theta 2$ ). This, together with (3.1), implies that $\theta(d(f u, f v)) \rightarrow 1$. Therefore $d(f u, f v) \rightarrow 0$ (due to $\Theta 2)$. Hence $f$ is continuous.

Remark 3.2 Observe that condition $\Theta 1$ can be withdrawn, and still Theorem 3.1 (also, most of the existence results in the literature (e.g., results of [25-29])) survives (in view of Proposition 3.1).

Now, Proposition 3.1 and Remark 3.2 led us to define a weaker contraction under the name of weak $\theta$-contraction as follows. 
Definition 3.2 Let $(M, d)$ be a metric space, and let $f: M \rightarrow M$. We say that $f$ is $a$ weak $\theta$-contraction if there exist $\theta \in \Theta_{2,3}$ (or $\left.\theta \in \Theta_{2,4}\right)$ and $h \in(0,1)$ such that (3.1) holds for all $u, v \in M$.

Remark 3.3 It is easy to verify that every Banach contraction is a weak $\theta$-contraction w.r.t. $\theta(\alpha)=e^{\alpha}\left(\right.$ or $\left.\theta(\alpha)=e^{\sqrt{\alpha}}\right)$ for all $\alpha>0$.

The following result shows that the completeness assumption of a metric space is a sufficient condition to show that a weak $\theta$-contraction is a Picard operator.

Theorem 3.2 Every weak $\theta$-contraction on a complete metric space is a Picard operator.

Proof If $\theta \in \Theta_{2,3}$, then, in view of Proposition 3.1, the proof runs along the lines of the proof of Theorem 3.1 (see [5, Thm. 2.1], wherein $\Theta 1$ is used only to show the continuity of $f$ ). Now, assume that $\theta \in \Theta_{2,4}$. Let $(M, d)$ be a complete metric space, and let $f: M \rightarrow M$ be a weak $\theta$-contraction. Let $u_{0} \in M$ be an arbitrary point. Define the Picard sequence $\left\{u_{n}\right\} \subseteq M$ by $u_{n+1}=f^{n+1} u_{0}=f u_{n}$ for all $n \in \mathbb{N}_{0}$. If there exists $n_{0} \in \mathbb{N}_{0}$ such that $u_{n_{0}}=f u_{n_{0}}$, then we are done. Assume that $u_{n} \neq u_{n+1}$ for all $n \in \mathbb{N}_{0}$. Applying (3.1), we have (for all $\left.n \in \mathbb{N}_{0}\right)$

$$
\theta\left(d\left(u_{n+1}, u_{n}\right)\right) \leq\left[\theta\left(d\left(u_{n}, u_{n-1}\right)\right)\right]^{h} \leq\left[\theta\left(d\left(u_{n-1}, u_{n-2}\right)\right)\right]^{h^{2}} \leq \cdots \leq\left[\theta\left(d\left(u_{1}, u_{0}\right)\right)\right]^{h^{n}} .
$$

Thus we have (for all $n \in \mathbb{N}_{0}$ )

$$
1<\theta\left(d\left(u_{n+1}, u_{n}\right)\right) \leq\left[\theta\left(d\left(u_{1}, u_{0}\right)\right)\right]^{h^{n}} .
$$

Letting $n \rightarrow \infty$, we have

$$
\lim _{n \rightarrow \infty} \theta\left(d\left(u_{n+1}, u_{n}\right)\right)=1 .
$$

Using $\Theta 2$, we obtain

$$
\lim _{n \rightarrow \infty} d\left(u_{n+1}, u_{n}\right)=0 .
$$

Now, we show that $\left\{u_{n}\right\}$ is a Cauchy sequence employing contradiction. To do so, assume that $\left\{u_{n}\right\}$ is not a Cauchy sequence. Then Lemma 2.1 and (3.2) ensure the existence of $\epsilon>0$ and two subsequences $\left\{u_{n(k)}\right\}$ and $\left\{u_{m(k)}\right\}$ of $\left\{u_{n}\right\}$ such that

$$
k \leq m(k)<n(k), \quad d\left(u_{n(k)-1}, u_{m(k)}\right)<\epsilon \leq d\left(u_{n(k)}, u_{m(k)}\right) \quad \forall k \geq 0
$$

and

$$
\lim _{k \rightarrow \infty} d\left(u_{n(k)}, u_{m(k)}\right)=\epsilon .
$$

Observe that

$$
\epsilon \leq d\left(u_{n(k)}, u_{m(k)}\right)
$$




$$
\begin{aligned}
& \leq d\left(u_{n(k)}, u_{(m(k)-1)}\right)+d\left(u_{m(k)-1}, u_{m(k)}\right) \\
& \leq d\left(u_{n(k)}, u_{(n(k)-1)}\right)+d\left(u_{n(k)-1}+u_{m(k)-1}\right)+d\left(u_{m(k)-1}, u_{m(k)}\right) \\
& \leq d\left(u_{n(k)}, u_{(n(k)-1)}\right)+d\left(u_{n(k)-1}+u_{m(k)}\right)+2 d\left(u_{m(k)-1}, u_{m(k)}\right) .
\end{aligned}
$$

Letting $k \rightarrow \infty$ and using (3.2), (3.3), and (3.4), we obtain

$$
\lim _{k \rightarrow \infty} d\left(u_{n(k)-1}, u_{m(k)-1}\right)=\epsilon
$$

It follows that there exists $N \in \mathbb{N}_{0}$ such that $d\left(u_{n(k)}, u_{m(k)}\right)>0$ for all $k \geq N$ (due to (3.4)). Applying (3.1), we have (for all $k \geq N$ )

$$
\theta\left(d\left(u_{n(k)}, u_{m(k)}\right)\right) \leq\left[\theta\left(d\left(u_{n(k)-1}, u_{m(k)-1}\right)\right)\right]^{h} .
$$

Letting $k \rightarrow \infty$ in (3.6) and using $\Theta 4$, (3.4), and (3.5), we obtain $\theta(\epsilon) \leq \theta(\epsilon)^{h}$, a contradiction. Hence $\left\{u_{n}\right\}$ is a Cauchy sequence. The completeness of $M$ implies that there exists $u \in M$ such that $\left\{u_{n}\right\}$ converges to $u$. The continuity of $f$ and the uniqueness of the limit give rise to $f u=u$. For the uniqueness part, assume by contradiction that $f$ has another fixed point, say $v \neq u$. Applying (3.1), we have $\theta(d(u, v)) \leq[\theta(d(u, v))]^{h}$, a contradiction. This concludes the proof.

Remark 3.4 In view of Remark 3.1, Theorem 3.2 with $\theta \in \Theta_{2,4}$ remains a weaker version of the main result of Piri and Kumam [23].

In the following example (inspired by [30]), we show that weak $\theta$-contractions are a proper generalization of Banach contractions.

Example 3.7 Let $M=[0, \infty)$ be endowed with the usual metric, and let $\beta \in M$. Define $f: M \rightarrow M$ by $f(u)=\frac{u}{i u+1}+\beta$, where $i \geq 1$. Consider the function $\theta:(0, \infty) \rightarrow(1, \infty)$ given in Example 3.4. Then

(a) $f$ is not a Banach contraction;

(b) $f$ is a weak $\theta$-contraction;

(c) $f$ is a Picard operator.

Proof (a) Define $u_{n}=\frac{1}{2 n}$ and $v_{n}=\frac{1}{n}$ for all $n \in \mathbb{N}$. Observe that

$$
\lim _{n \rightarrow \infty} \frac{\left|f u_{n}-f v_{n}\right|}{\left|u_{n}-v_{n}\right|}=\lim _{n \rightarrow \infty} \frac{\left|\frac{u_{n}}{i u_{n}+1}-\frac{v_{n}}{i v_{n}+1}\right|}{\left|u_{n}-v_{n}\right|}=\lim _{n \rightarrow \infty} \frac{1}{\left(\frac{i}{2 n}+1\right)\left(\frac{i}{n}+1\right)}=1 .
$$

Thus there is no $h \in(0,1)$ such that $d(f u, f v) \leq h d(u, v)$ for all $u, v \in M$. Hence $f$ is not a Banach contraction.

(b) To prove (3.1), it is equivalent to show that (for all $u, v \in M$ with $f u \neq f v$ )

$$
d(f u, f v) e^{\frac{-1}{d(u, f v)}} \leq h d(u, v) e^{\frac{-1}{d(u, v)}} \quad \text { for some } h \in(0,1),
$$

or

$$
\ln (d(f u, f v))-\frac{1}{d(f u, f v)} \leq \ln h+\ln (d(u, v))-\frac{1}{d(u, v)} \quad \text { for some } h \in(0,1) .
$$


Now, observe that (for all $u, v \in M$ with $f u \neq f v$ and $u>v$ )

$$
\begin{aligned}
& \ln (d(f u, f v))-\frac{1}{d(f u, f v)}-\ln (d(u, v))-\frac{1}{d(u, v)} \\
& \quad=\ln \left(\frac{u}{i u+1}-\frac{v}{i v+1}\right)-\left(\frac{u}{i u+1}-\frac{v}{i v+1}\right)^{-1}-\ln (u-v)+\frac{1}{u-v} \\
& \quad=-\ln ((i u+1)(i v+1))+\frac{1-(i u+1)(i v+1)}{u-v} \\
& \quad \leq-\frac{i^{2} u v+i u+i v}{u-v} \\
& \quad \leq-i \\
& \leq-1
\end{aligned}
$$

The case $u<v$ can be treated analogously. Hence (3.7) holds for any $h \in(0,1)$ with $\ln h \geq$ -1 . Therefore, $f$ is a weak $\theta$-contraction.

(c) Follows immediately from Theorem 3.2 as $(M, d)$ is a complete metric space and in view of (b). The fixed point of $f$ is $z=\frac{\beta i+\sqrt{\beta^{2} i^{2}+4 \beta i}}{2 i}$.

Theorem 3.2 can be improved as follows.

Theorem 3.3 Let $(M, d)$ be a complete metric space, and let $f: M \rightarrow M$. If there exists $n \in \mathbb{N}$ such that $f^{n}$ is a weak $\theta$-contraction, then $f$ is a Picard operator.

Proof Theorem 3.2 ensures that $f^{n}$ is a Picard operator, so that there exists a unique $z \in M$ such that $f^{n} z=z$ and $\lim _{m \rightarrow \infty}\left(f^{n} u\right)^{m}=z$ for all $u \in M$. Observe that $f^{n+1} z=f z$. Thus $f z$ is also a fixed point of $f^{n}$. Therefore $f z=z$. Moreover, if $z^{*}$ is another fixed point of $f$, then it is also a fixed point of $f^{n}$. Hence $z=z^{*}$. Thus $f$ has a unique fixed point.

Now, let $m$ be a positive integer greater than $n$. Then there exist $l \geq 1$ and $s \in$ $\{0,1,2, \ldots, n-1\}$ such that $m=n l+s$. Notice that (for all $u \in M$ )

$$
\lim _{m \rightarrow \infty} f^{m} u=\lim _{l \rightarrow \infty} f^{n l}\left(f^{s} u\right)=z
$$

This concludes the proof.

\section{Application: weak $\boldsymbol{\theta}$-iterated function systems}

In this section, we apply our results to obtain the existence and uniqueness of the attractors of some iterated function systems composed by weak $\theta$-contractions on a complete metric space. In the following, $(M, d)$ is a complete metric space, $N \in \mathbb{N}$, and $\theta \in \Theta_{1,2,4}$.

Definition 4.1 Let $\left\{f_{i}\right\}_{i=1}^{N}$ be a finite family of self-mappings on $M$. If (for each $i$ ) $f_{i}: M \rightarrow$ $M$ is a weak $\theta$-contraction, then the family $\left\{f_{i}\right\}_{i=1}^{N}$ is called $a$ weak $\theta$-iterated function system (weak $\theta$-IFS).

The set function $\mathcal{G}: \mathcal{K}(M) \rightarrow \mathcal{K}(M)$ defined by $\mathcal{G}(B)=\bigcup_{i=1}^{N} f_{i}(B)$ (for all $B \in \mathcal{K}(M)$ ) is called the associated Hutchinson operator. A set $A \in \mathcal{K}(M)$ is called an attractor of the weak $\theta$-IFS if $\mathcal{G}(A)=A$. 
Now, we prove that the weak $\theta$-IFS has a unique attractor. To do so, we begin with the following:

Lemma 4.1 Let $f: M \rightarrow M$ be a weak $\theta$-contraction. Then the mapping $A \longmapsto f(A)$ is also a weak $\theta$-contraction from $\mathcal{K}(M)$ into itself.

Proof Let $A, B \in \mathcal{K}(M)$ be such that $\eta(f(A), f(B))>0$. Assume that

$$
\eta(f(A), f(B))=D(f(A), f(B))=\sup _{u \in A} \inf _{v \in B} d(f u, f v)>0 .
$$

As $f$ is a weak $\theta$-contraction, there exists $h \in(0,1)$ such that $\theta(d(f u, f v)) \leq[\theta(d(u, v))]^{h}$ for all $u, v \in M$ with $f u \neq f v$. Now, using (4.1), the compactness of $A$, and the continuity of $f$, we can find $a \in A$ such that $D(f(A), f(B))=\inf _{v \in B} d(f a, f v)>0$, so that $d(f a, f v)>0$ for all $v \in B$. Hence

$$
\theta\left(\inf _{v \in B} d(f a, f v)\right) \leq \theta(d(f a, f v)) \leq[\theta(d(a, v))]^{h} \quad \text { for all } v \in B
$$

Thus

$$
\theta(\eta(f(A), f(B))) \leq[\theta(d(a, v))]^{h} \quad \text { for all } v \in B
$$

Now, let $b \in B$ be such that $d(a, b)=\inf _{v \in B} d(a, v)$. From (4.2) we have

$$
\begin{aligned}
\theta(\eta(f(A), f(B))) & \leq[\theta(d(a, b))]^{h} \\
& =\left[\theta\left(\inf _{v \in B} d(a, v)\right)\right]^{h} \\
& \leq\left[\theta\left(\sup _{u \in A} \inf _{v \in B} d(u, v)\right)\right]^{h} \\
& =[\theta(D(A, B))]^{h} \\
& \leq[\theta(\eta(A, B))]^{h} .
\end{aligned}
$$

This completes the proof.

Now, we can state and prove our main result in this section.

Theorem 4.1 If $\left\{f_{i}\right\}_{i=1}^{N}$ is a weak $\theta$-IFS, then it has a unique attractor $A$. Moreover, $A=$ $\lim _{n \rightarrow \infty} \mathcal{G}^{n}(B)$ for all $B \in \mathcal{K}(M)$, the limit being taken w.r.t. the Hausdorff-Pompeiu metric.

Proof For each $i \in\{1,2, \ldots, N\}$, let $h_{i}$ be the constant given by (3.1), which is associated with $f_{i}$. Let $B, C \in \mathcal{K}(M)$ be such that $\eta(\mathcal{G}(B), \mathcal{G}(C))>0$. Now, Lemma 2.3 implies that

$$
0<\eta(\mathcal{G}(B), \mathcal{G}(C)) \leq \sup _{1 \leq i \leq N} \eta\left(f_{i}(B), f_{i}(C)\right)=\eta\left(f_{i_{0}}(B), f_{i_{0}}(C)\right)
$$

for some $i_{0} \in\{1,2, \ldots, N\}$. Using $\Theta 1$ and Lemma 4.1, we have

$$
\theta(\eta(\mathcal{G}(B), \mathcal{G}(C))) \leq \theta\left(\eta\left(f_{i_{0}}(B), f_{i_{0}}(C)\right)\right) \leq[\theta(\eta(B, C))]^{h_{i_{0}}} .
$$


Therefore $\mathcal{G}$ is a weak $\theta$-contraction on the complete metric space $(\mathcal{K}(M), \eta)$. Hence Theorem 3.2 ensures the existence and uniqueness of $A \in \mathcal{K}(M)$ such that $\mathcal{G}(A)=A$ and $A=\lim _{n \rightarrow \infty} \mathcal{G}^{n}(B)$ for all $B \in \mathcal{K}(M)$, as required.

In support of Theorem 4.1, we provide the following:

Example 4.1 Let $M=[0, \infty)$ be endowed with the usual metric, $i \in\{1,2, \ldots, N\}$, and $\beta_{i} \in$ $M$ (for all $i$ ). Define $f_{i}: M \rightarrow M$ by: $f_{i}(u)=\frac{u}{i u+1}+\beta_{i}$. Consider the function $\theta:(0, \infty) \rightarrow$ $(1, \infty)$ given in Example 3.4. Then $\left\{f_{i}\right\}_{i=1}^{N}$ is a weak $\theta$-IFS and has a unique attractor, which is approximated (w.r.t. the Hausdorf-Pompeiu metric) by the sequence $\left\{\mathcal{G}^{n}(B)\right\}$ for all $B \in \mathcal{K}(M)$. Furthermore $\left\{f_{i}\right\}_{i=1}^{N}$ is not a classical Hutchinson IFS.

Proof In view of Example 3.7, we conclude that $f_{i}, i \in\{1,2, \ldots, N\}$, are weak $\theta$-contractions and are not Banach contractions. Hence, the conclusion follows immediately from Theorem 4.1.

\section{Relation-theoretic fixed point results}

Let $M$ be a nonempty set, and let $u, v \in M$. A subset $\mathcal{R}$ of $M \times M$ is called a binary relation on M. If $(u, v) \in \mathcal{R}$, then we write $u \mathcal{R} v$. Two elements $u, v \in M$ are said to be comparable under $\mathcal{R}$ if either $u \mathcal{R} v$ or $v \mathcal{R} u$, which is often denoted by $[u, v] \in \mathcal{R}$. A binary relation $\mathcal{R}$ is said to be: reflexive if $u \mathcal{R} u$ for any $u \in M$; transitive if for any $u, v, z \in M, u \mathcal{R} v$ and $v \mathcal{R} z$ imply $u \mathcal{R} z$; antisymmetric if for any $u, v \in M, u \mathcal{R} v$ and $v \mathcal{R} u$ imply $u=v$; partial order if it is reflexive, transitive, and antisymmetric. If $f: M \rightarrow M$, then $\mathcal{R}$ is said to be $f$-transitive if it is transitive on $f(M)$.

In the following results, $\theta:(0, \infty] \rightarrow(1, \infty]$ is such that $\theta(\alpha)=\infty$ if and only if $\alpha=\infty$. Now, we can state and prove our results in this section.

Theorem 5.1 Let $(M, d, \mathcal{R})$ be a generalized metric space endowed with binary relation, $u_{0} \in M$, and $f: M \rightarrow M$. Suppose that the following conditions are satisfied:

(a) $u_{0} \mathcal{R} f u_{0}$, and $\mathcal{R}$ is $f$-transitive;

(b) for any $u, v \in M, u \mathcal{R} v$ implies fuR $f v$;

(c) every Cauchy sequence $\left\{u_{n}\right\} \subseteq M$ with $u_{n} \mathcal{R} u_{n+1}$ converges to some $u \in M$;

(d) there exist $\theta \in \Theta_{2,3}$ and $h \in(0,1)$ such that (3.1) holds for all $u, v \in M$ with $u \mathcal{R} v$ and $d(u, v)<\infty$;

(e) $f$ is continuous.

Then one of the following assertions holds:

(I) $d\left(f^{n} u_{0}, f^{n+1} u_{0}\right)=\infty$ for all $n \in \mathbb{N}$; or

(II) $f$ has a fixed point that is approximated by $\left\{f^{n} u_{0}\right\}$.

Proof Define a sequence $\left\{u_{n}\right\}$ of Picard iterates based on $u_{0}$, that is, $u_{n}=f^{n} u_{0}$. Consider the sequence $\left\{d\left(u_{n}, u_{n+1}\right)\right\}$ of nonnegative real numbers. There are two mutually exclusive possibilities:

(i) for every $n \in \mathbb{N}, d\left(u_{n}, u_{n+1}\right)=\infty$, which is precisely the alternative (I) of the conclusion of the theorem; or

(ii) there exists $n \in \mathbb{N}$ such that $d\left(u_{n}, u_{n+1}\right)<\infty$; in such a case, we will show that conclusion (II) of the theorem is fulfilled. 
If case (ii) holds, then let $p$ be the smallest integer satisfying (ii). If $d\left(u_{n}, u_{n+1}\right)=0$ for some $n \geq p$, then we are done. Suppose that $d\left(u_{n}, u_{n+1}\right)>0$ for all $n \geq p$. Applying condition (d), we have $\theta\left(d\left(f u_{p}, f u_{p+1}\right)\right) \leq\left[\theta\left(u_{p}, u_{p+1}\right)\right]^{h}<\theta\left(d\left(u_{p}, u_{p+1}\right)\right)<\infty$, which implies $d\left(u_{p+1}, u_{p+2}\right)<\infty$. Hence by mathematical induction we have $d\left(u_{p+n}, u_{p+n+1}\right)<\infty$ for all $n \in \mathbb{N}$. In other words, $d\left(u_{n}, u_{n+1}\right)<\infty$ for all $n \geq p$. Now, as $u_{0} \mathcal{R} f u_{0}$, using assumption (b), we have $u_{n} \mathcal{R} u_{n+1}$ for all $n \geq 0$. Applying assumption (d), we obtain (for all $n \geq p$ )

$$
\theta\left(d\left(u_{n}, u_{n+1}\right)\right) \leq\left[\theta\left(d\left(u_{n-1}, u_{n}\right)\right)\right]^{h} \leq\left[\theta\left(d\left(u_{n-2}, u_{n-1}\right)\right)\right]^{h^{2}} \leq \cdots \leq\left[\theta\left(d\left(u_{p}, u_{p+1}\right)\right)\right]^{h^{n-p}} .
$$

Thus we have (for all $n \geq p$ )

$$
1<\theta\left(d\left(u_{n}, u_{n+1}\right)\right) \leq\left[\theta\left(d\left(u_{p}, u_{p+1}\right)\right)\right]^{h^{n-p}} .
$$

Letting $n \rightarrow \infty$ in (5.1), we obtain $\lim _{n \rightarrow \infty} \theta\left(d\left(u_{n}, u_{n+1}\right)\right)=1$, which (in view of $\Theta 2$ ) implies that

$$
\lim _{n \rightarrow \infty} d\left(u_{n}, u_{n+1}\right)=0
$$

Now, by $\Theta 3$ there exist $r \in(0,1)$ and $0<l \leq \infty$ such that

$$
\lim _{n \rightarrow \infty} \frac{\theta\left(d\left(u_{n}, u_{n+1}\right)\right)-1}{\left[d\left(u_{n}, u_{n+1}\right)\right]^{r}}=l .
$$

Two cases can occur depending on $l$. Firstly, assume that $l<\infty$. Let $A=\frac{l}{2}$. By the definition of the limit there exists a positive integer $N_{0}>p$ such that

$$
\left|\frac{\theta\left(d\left(u_{n}, u_{n+1}\right)\right)-1}{\left[d\left(u_{n}, u_{n+1}\right)\right]^{r}}-l\right| \leq A \quad \text { for all } n \geq N_{0}
$$

which implies

$$
\frac{\theta\left(d\left(u_{n}, u_{n+1}\right)\right)-1}{\left[d\left(u_{n}, u_{n+1}\right)\right]^{r}} \geq l-A=A \quad \text { for all } n \geq N_{0},
$$

and so

$$
n\left[d\left(u_{n}, u_{n+1}\right)\right]^{r} \leq n A^{*}\left[\theta\left(d\left(u_{n}, u_{n+1}\right)\right)-1\right] \text { for all } n \geq N_{0},
$$

where $A^{*}=\frac{1}{A}$. Secondly, if $l=\infty$, then let $B>0$ be a given real number. Again, the definition of the limit implies that there exists a positive integer $N_{1}>p$ such that

$$
\frac{\theta\left(d\left(u_{n}, u_{n+1}\right)\right)-1}{\left[d\left(u_{n}, u_{n+1}\right)\right]^{r}} \geq B \quad \text { for all } n \geq N_{1},
$$

which implies

$$
n\left[d\left(u_{n}, u_{n+1}\right)\right]^{r} \leq n B^{*}\left[\theta\left(d\left(u_{n}, u_{n+1}\right)\right)-1\right] \text { for all } n \geq N_{1},
$$


where $B^{*}=\frac{1}{B}$. Therefore, in all cases, there exist a real constant $C>0$ and a positive integer $N_{2}>p$ such that

$$
n\left[d\left(u_{n}, u_{n+1}\right)\right]^{r} \leq n C\left[\theta\left(d\left(u_{n}, u_{n+1}\right)\right)-1\right] \text { for all } n \geq N_{2} .
$$

Using (d), we have

$$
n\left[d\left(u_{n}, u_{n+1}\right)\right]^{r} \leq n C\left(\left[\theta\left(d\left(u_{p}, u_{p+1}\right)\right)\right]^{k^{n-p}}-1\right) \quad \text { for all } n \geq N_{2} .
$$

Letting $n \rightarrow \infty$ in the last inequality, we have

$$
\lim _{n \rightarrow \infty} n\left[d\left(u_{n}, u_{n+1}\right)\right]^{r}=0 .
$$

Hence, there exists a positive integer $N_{3}>p$ such that

$$
d\left(u_{n}, u_{n+1}\right) \leq \frac{1}{n^{\frac{1}{r}}} \quad \text { for all } n \geq N_{3}
$$

Now, for $m>n>N_{3}$, we have

$$
d\left(u_{n}, u_{m}\right) \leq \sum_{i=n}^{m-1} d\left(u_{n}, u_{n+1}\right) \leq \sum_{i=n}^{m-1} \frac{1}{i^{\frac{1}{r}}} .
$$

The convergence of the series $\sum_{i=n}^{\infty} \frac{1}{i^{\frac{1}{r}}}\left(\right.$ as $\left.\frac{1}{r}>1\right)$ implies that $\left\{u_{n}\right\}_{n \geq p}$ is a Cauchy sequence and hence converges to some $u \in M$ (due to condition (c)). The continuity of $f$ and the uniqueness of the limit give $f u=u$. This concludes the proof.

Now, we give the following example, which exhibits the utility of Theorem 5.1.

Example 5.1 Let $M=(0, \infty)$ be endowed with the usual metric. Then $d(u, v)<\infty$ for all $u, v \in M$, and hence alternative (I) of Theorem 5.1 is excluded. Consider the sequence $\left\{\pi_{n}\right\}$ in $M$ defined by

$$
\pi_{n}=\frac{(n+1)(n+2)}{2} \text { for all } n \in \mathbb{N}
$$

Define the binary relation $\mathcal{R}$ as follows:

$$
\mathcal{R}=\left\{\left(\pi_{1}, \pi_{1}\right)\right\} \cup\left\{\left(\pi_{n}, \pi_{n+1}\right): n \in \mathbb{N}\right\} .
$$

Observe that $M$ satisfies condition (c) of Theorem 5.1. Now, define the mapping $f: M \rightarrow$ $M$ by

$$
f u= \begin{cases}u & \text { if } 0<u \leq \pi_{1} \\ \pi_{1} & \text { if } \pi_{1} \leq u \leq \pi_{2} \\ \pi_{i}+\left(\frac{\pi_{i+1}-\pi_{i}}{\pi_{i+2}-\pi_{i+1}}\right)\left(u-\pi_{i+1}\right) & \text { if } \pi_{i+1} \leq u \leq \pi_{i+2}, i=1,2, \ldots\end{cases}
$$


Then $f$ is continuous, $\pi_{1} \mathcal{R} f \pi_{1}$, and $\mathcal{R}$ is $f$-transitive and satisfies condition (b). Now, we will show that $f$ satisfies condition (d) with the function $\theta:(0, \infty] \rightarrow(1, \infty]$ defined by $\theta(\alpha)=e^{\sqrt{\alpha e^{\alpha}}}$ for $\alpha>0$. It easy to show that $\theta \in \Theta_{2,3}$ and $\theta(\alpha)=\infty$ if and only if $\alpha=\infty$. Now, let $u, v \in M$ be such that $u \mathcal{R} v$ and $f u \neq f v$. It must be the case $u=\pi_{n}$ and $v=\pi_{n+1}$ for some $n \in \mathbb{N}$. To prove condition (3.1), it is enough to show that

$$
d(f u, f v) e^{d(f u, f v)} \leq h^{2} d(u, v) e^{d(u, v)}
$$

for some $h \in(0,1)$, that is, we have to show that

$$
\frac{d(f u, f v)}{d(u, v)} e^{d(f u, f v)-d(u, v)} \leq h^{2}
$$

for some $h \in(0,1)$. Now, observe that

$$
\frac{d(f u, f v)}{d(u, v)} e^{d(f u, f v)-d(u, v)}=\frac{\pi_{n}-\pi_{n-1}}{\pi_{n+1}-\pi_{n}} e^{\left(\pi_{n}-\pi_{n-1}\right)-\left(\pi_{n+1}-\pi_{n}\right)}<e^{-1} .
$$

Hence inequality (5.4) holds for $h=e^{\frac{-1}{2}}$. Therefore, all the hypotheses of Theorem 5.1 (alternative (II)) are satisfied. Observe that $f$ has infinitely many fixed points $(\operatorname{Fix}(f)=$ $(0,3])$.

\section{Remark 5.1}

1 Theorems 3.1 and 3.2 are not applicable in the context of Example 5.1 as condition

(3.1) does not hold on $(0,3]$ and also $(M, d)$ is not a complete space.

2 Theorems of $[14,22,31]$ do not work in the context of Example 5.1 as $\lim _{n \rightarrow \infty} \frac{d\left(f \pi_{n} f 1\right)}{d\left(\pi_{n}, 1\right)}=1$, so that their contraction conditions do not hold.

Next, we present an analogue of Theorem 5.1 avoiding the continuity assumption of $f$.

Theorem 5.2 The conclusions of Theorem 5.1 remain true if condition (e) is replaced by the following one:

$\left(\mathrm{e}^{\prime}\right)$ for every convergent sequence $\left\{u_{n}\right\} \subseteq M$ and all $u \in M$,

$$
\left[u_{n} \mathcal{R} u_{n+1} \text { and }\left\{u_{n}\right\} \rightarrow u\right] \Rightarrow\left[u_{n} \mathcal{R} u \text { for all } n \in \mathbb{N}\right]
$$

Proof Following the proof of Theorem 5.1, we have that $\left\{u_{n}\right\}_{n \geq p}$ is a convergent sequence with $u_{n} \mathcal{R} u_{n+1}$ (for all $n$ ) and converges to $u \in M$. From our assumption we have $u_{n} \mathcal{R} u$ (for all $n$ ). Let $P=\left\{n \geq p: f u_{n}=f u\right\}$. If $P$ is an infinite set, then $\left\{u_{n}\right\}_{n \geq p}$ has a subsequence converging to $f u$. The uniqueness of the limit gives $f u=u$. Now, assume that $P$ is finite. Then $\left\{u_{n}\right\}_{n \geq p}$ has a subsequence $\left\{u_{n_{k}}\right\}$ such that $f u_{n_{k}} \neq f u$ for all $k \in \mathbb{N}$. Observe that $\lim _{k \rightarrow \infty} d\left(u_{n_{k}}, u\right)=0$ implies that $d\left(u_{n_{k}}, u\right)<\infty$ for all $k \in \mathbb{N}$. Now, applying (3.1), we have

$$
\theta\left(d\left(f u_{n_{k}}, f u\right)\right) \leq\left[\theta\left(d\left(u_{n_{k}}, u\right)\right)\right]^{h} \text { for all } k \in \mathbb{N}
$$

Letting $k \rightarrow \infty$ and using $\Theta 2$, we obtain $\lim _{k \rightarrow \infty} d\left(f u_{n_{k}}, f u\right)=0$. Again the uniqueness of the limit implies $f u=u$. This concludes the proof. 
The following example exhibits the utility of Theorem 5.2.

Example 5.2 Let $M=(-1,3]$ be equipped with the usual metric. Define the binary relation $\mathcal{R}$ on $M$ as follows:

$$
\mathcal{R}=\{(0,0),(0,1),(1,0),(1,1),(0,3)\}
$$

Also, define $f: M \rightarrow M$ by

$$
f u= \begin{cases}0 & \text { if }-1<u \leq 1 \\ 1 & \text { if } 1<u \leq 3\end{cases}
$$

Observe that $f$ is not continuous. Now, we have the following:

- $d(u, v)<\infty$ for all $u, v \in M$, so that alternative (I) of Theorem 5.2 is excluded;

- $0 \in M$ and $0 \mathcal{R} f 0($ as $(0,0) \in \mathcal{R})$;

- $\mathcal{R}$ is $f$-transitive (as $\mathcal{R}$ is transitive on $\{0,1\}$ );

- for any $u, v \in M, u \mathcal{R} v$ implies $f u \mathcal{R} f v$;

- if $\left\{u_{n}\right\}$ is a Cauchy sequence in $M$ with $u_{n} \mathcal{R} u_{n+1}$, then there exists $N \in \mathbb{N}$ such that either $u_{n}=0$ for all $n \geq N$ or $u_{n}=1$ for all $n \geq N$ so that $\left\{u_{n}\right\}$ converges to either 0 or 1 , which are in $M$;

- $f$ satisfies (3.1) for all $u, v \in M$ with $u \mathcal{R} v$ and $f u \neq f v$ (namely, for $u, v \in\{0,3\}$ ) with $\theta$ given in Example 3.1 and any $h \in\left[\frac{1}{2}, 1\right)$;

- if $\left\{u_{n}\right\}$ is a sequence in $M$ such that $u_{n} \mathcal{R} u_{n+1}$, then we may observe that $\left(u_{n}, u_{n+1}\right) \notin\{(0,3)\}$, so that $\left(u_{n}, u_{n+1}\right) \in\{(0,0),(0,1),(1,0),(1,1)\}$, and hence $\left\{u_{n}\right\} \subset\{0,1\}$, which is closed, so that $u_{n} \mathcal{R} u$ for all $n$.

Thus all the assumptions of Theorem 5.2 (alternative (II)) are satisfied ensuring the existence of a fixed point of $f$ (namely $u=0$ ).

Now, we present a corresponding uniqueness result as follows.

Theorem 5.3 If in addition to the hypotheses of Theorem 5.1 (or Theorem 5.2), we assume that, for each $u, v \in \operatorname{Fix}(f)$, there exists $z_{0} \in M$ comparable to both $u$ and $v, d\left(u, z_{0}\right)<\infty$, and $d\left(v, z_{0}\right)<\infty$, then $f$ is a Picard operator.

Proof In view of Theorem 5.1 (or Theorem 5.2), the set Fix $(f)$ is nonempty. Let $u, v \in$ $\operatorname{Fix}(f)$. By our assumption there exists $z_{0} \in M$ such that $\left[u, z_{0}\right] \in \mathcal{R}$ and $\left[v, z_{0}\right] \in \mathcal{R}$. Let $\left\{z_{n}\right\}$ be the Picard sequence under $f$ based on $z_{0}$, that is, $z_{n}=f^{n} z_{0}$ for all $n \geq 0$. Now, we show that $u=v$ by proving $\left\{z_{n}\right\} \rightarrow u$ and $\left\{z_{n}\right\} \rightarrow v$.

As $\left[u, z_{0}\right] \in \mathcal{R}$, either $u \mathcal{R} z_{0}$ or $z_{0} \mathcal{R} u$. Assume that $u \mathcal{R} z_{0}$. If $u=z_{n_{0}}$ for some $n_{0} \geq 0$, then $u=z_{n}$ for all $n \geq n_{0}$. Thus $\left\{z_{n}\right\} \rightarrow u$. Assume that $u \neq z_{n}$ for all $n \geq 0$. Using condition (b), we have $u \mathcal{R} z_{n}$ for all $n \geq 0$. Observe that $\theta\left(d\left(u, z_{1}\right)\right) \leq\left[\theta\left(d\left(u, z_{0}\right)\right)\right]^{h}<\infty$ implies $d\left(u, z_{1}\right)<$ $\infty$, so that by induction we have $d\left(u, z_{n}\right)<\infty$ for all $n \geq 0$. Now, applying condition (d), we have (for all $n \geq 0$ )

$$
\theta\left(d\left(u, z_{n}\right)\right) \leq\left[\theta\left(d\left(u, z_{n-1}\right)\right)\right]^{h} \leq\left[\theta\left(d\left(u, z_{n-2}\right)\right)\right]^{h^{2}} \leq \cdots \leq\left[\theta\left(d\left(u, z_{0}\right)\right)\right]^{h^{n}} .
$$


Thus, we have (for all $n \geq 0$ )

$$
1<\theta\left(d\left(u, z_{n}\right)\right) \leq\left[\theta\left(d\left(u, z_{0}\right)\right)\right]^{h^{n}} .
$$

Letting $n \rightarrow \infty$ in (5.5), we obtain $\lim _{n \rightarrow \infty} \theta\left(d\left(u, z_{n}\right)\right)=1$, which (in view of $\Theta 2$ ) implies that $\lim _{n \rightarrow \infty} d\left(u, z_{n}\right)=0$. The proof of the case $z_{0} \mathcal{R} u$ is similar. Similarly, we can prove that $\left\{z_{n}\right\} \rightarrow v$. This concludes the proof.

The following example exhibits the utility of Theorem 5.3.

Example 5.3 Let $M=[3, \infty)$ be endowed with the usual metric and define $f: M \rightarrow M$ by

$$
f u= \begin{cases}3 & \text { if } 3 \leq u \leq \pi_{2}, \\ \pi_{i}+\left(\frac{\pi_{i+1}-\pi_{i}}{\pi_{i+2}-\pi_{i+1}}\right)\left(u-\pi_{i+1}\right) & \text { if } \pi_{i+1} \leq u \leq \pi_{i+2}, i=1,2, \ldots\end{cases}
$$

If we set out the rest as in Example 5.1, then all the hypotheses of Theorem 5.3 are satisfied, and $f$ has a unique fixed point (namely $u=3$ ).

Setting $\mathcal{R}=M \times M$ in Theorem 5.3, we deduce the following corollary.

Corollary 5.1 Let $(M, d)$ be a generalized complete metric space, and let $f: M \rightarrow M$. If there exist $\theta \in \Theta_{2,3}$ and $h \in(0,1)$ such that (3.1) holds for all $u, v \in M$ with $d(u, v)<\infty$, then $f$ is a Picard operator.

Remark 5.2 Corollary 5.1 remains a sharpened version of Theorem 3.1 due to Jleli and Samet [5].

\section{Application: weak $\boldsymbol{\theta}$-countable iterated function systems}

In this section, inspired by [30], we apply our results to obtain the existence and uniqueness of the attractors of some countable iterated function systems composed by weak $\theta$ contractions on a generalized complete metric space. In the following, $(M, d)$ is a complete metric space, $C L(M)$ is the class of all nonempty closed subsets of $M$, and $\theta \in \Theta_{1,2,3}$.

Definition 6.1 Let $\left\{f_{i}\right\}_{i \geq 1}$ be a countable family of self-mappings on $M$. If (for each $i$ ) $f_{i}: M \rightarrow M$ is a weak $\theta$-contraction, then the countable family $\left\{f_{i}\right\}_{i \geq 1}$ is called $a$ weak $\theta$ countable iterated function system (weak $\theta$-CIFS).

The set function $\mathcal{G}: C L(M) \rightarrow C L(M)$ defined by $\mathcal{G}(B)=\overline{\bigcup_{i \geq 1} f_{i}(B)}$ (for all $B \in C L(M)$ ) is called the associated Hutchinson operator. A set $A \in C L(M)$ is called an attractor of the weak $\theta-\operatorname{CIFS}$ if $\mathcal{G}(A)=A$.

Before giving our main result in this section, we prove the following lemma.

Lemma 6.1 Assume that $\theta$ is continuous and $f: M \rightarrow M$ is a weak $\theta$-contraction with constant from (3.1) equal to $h^{2}$. Then

$$
\theta(\eta(f(B), f(C))) \leq[\theta(\eta(B, C))]^{h}
$$

for all $B, C \in C L(M)$ with $C \subset B$ or $B \subset C, \eta(B, C)<\infty$, and $f(B) \neq f(C)$. 
Proof Let $B, C \in C L(M)$ with $C \subset B, \eta(B, C)<\infty$, and $f(B) \neq f(C)$. Then $f(C) \subset f(B)$, $D(B, C)<\infty$, and $D(C, B)=0$ (in view of Lemma 2.2). Now, if $f$ is a weak $\theta$-contraction for $\theta \in \Theta_{1,2,3}$, then $d(f u, f v) \leq d(u, v)$ for all $u \in B, v \in C$. This implies that

$$
D(f(B), f(C))=\sup _{u \in B} \inf _{v \in C} d(f u, f v) \leq \sup _{u \in B} \inf _{v \in C} d(u, v)=D(B, C)<\infty .
$$

Observe that $\eta(f(B), f(C))>0$ and $D(f(C), f(B))=0$ imply that $D(f(B), f(C))>0$. Now, if $\theta:(0, \infty) \rightarrow(1, \infty)$ is a continuous mapping, then $\theta^{*}:(0, \infty) \rightarrow(-\infty, \infty)$ given by $\theta^{*}(\alpha)=$ $\ln \ln \theta(\alpha)$ for all $\alpha>0$ is also a continuous mapping. By the continuity of $\theta^{*}$, for $-\ln h>0$, there exists a positive $\epsilon_{0}<D(f(B), f(C))$ such that

$$
\begin{gathered}
\alpha \in\left(D(f(B), f(C))-\epsilon_{0}, D(f(B), f(C))+\epsilon_{0}\right) \\
\Rightarrow \quad \theta^{*}(\alpha)>\theta^{*}(D(f(B), f(C)))+\ln h .
\end{gathered}
$$

Now, we can find $b \in B$ such that $\inf _{v \in C} d(f b, f v)+\epsilon_{0}>D(f(B), f(C))$. Observe that $f(b) \notin$ $f(C)$ since otherwise $\epsilon_{0}>D(f(B), f(C))$, which contradicts the choice of $b$. Putting $\alpha=$ $\inf _{v \in C} d(f b, f v)$ in (6.1), we obtain

$$
\theta^{*}(D(f(B), f(C)))<-\ln h+\theta^{*}\left(\inf _{v \in C} d(f b, f v)\right)
$$

Now, by the hypothesis we have $\theta(d(f u, f v)) \leq[\theta(d(u, v))]^{h^{2}}$ for all $u \in B$ and $v \in C$ with $f u \neq$ $f v$, that is, $\theta^{*}(d(f u, f v)) \leq 2 \ln h+\theta^{*}(d(u, v))$ for all $u \in B$ and $v \in C$ with $f u \neq f v$. Therefore, using (6.2) and the fact that $\ln h<0$, we have

$$
\begin{aligned}
-\ln h+\theta^{*}(D(f(B), f(C))) & <-2 \ln h+\theta^{*}\left(\inf _{v \in C} d(f b, f v)\right) \\
& \leq-2 \ln h+\theta^{*}(d(f b, f v)) \\
& \leq \theta^{*}(d(b, v))
\end{aligned}
$$

for all $v \in C$. Accordingly, using the continuity of $\theta^{*}$, we have

$$
-\ln h+\theta^{*}(D(f(B), f(C))) \leq \theta^{*}\left(\inf _{v \in C} d(b, v)\right) \leq \theta^{*}(D(B, C))=\theta^{*}(\eta(B, C)) .
$$

Now, as $D(f(C), f(B))=0$, we have

$$
-\ln h+\theta^{*}(\eta(f(B), f(C))) \leq \theta^{*}(\eta(B, C))
$$

or

$$
\ln \ln \theta(\eta(f(B), f(C))) \leq \ln h+\ln \ln \theta(\eta(B, C)),
$$

which implies that

$$
\theta(\eta(f(B), f(C))) \leq[\theta(\eta(B, C))]^{h} .
$$


This completes the lemma.

Now, we can present our main result of this section.

Theorem 6.1 Let $\left\{f_{i}\right\}_{i \geq 1}$ be a weak $\theta$-CIFS on the complete metric space $(M, d)$, and let $\mathcal{G}: C L(M) \rightarrow C L(M)$ be defined as in Definition 6.1. Assume that $\theta$ is continuous and $h_{i}$ is the constant given by (3.1) associated with $f_{i}$ for each $i \geq 1$. If $h=\sup _{i \geq 1} h_{i}<1$ and for every nonempty $K \subset \mathbb{N}$, we put $C_{K}=\overline{\left\{z_{i}: i \in K\right\}}$, where $z_{i}$ is the fixed point of $f_{i}$, then one of the following assertions holds:

(i) $\eta\left(\mathcal{G}^{n}\left(C_{K}\right), \mathcal{G}^{n+1}\left(C_{K}\right)\right)=\infty$ for all $n \in \mathbb{N}$, or

(ii) there exists an attractor $A \in C L(M)$ of the considered weak $\theta$-CIFS and $A=\lim _{n \rightarrow \infty} \mathcal{G}^{n}\left(C_{K}\right)$, the limit being taken w.r.t. the generalized Hausdorff-Pompeiu metric.

Moreover, if $\mathcal{G}(M)$ is bounded or, in particular, $\mathcal{G}(M)$ is compact, then the attractor $A$ is unique and approximated by the sequence $\left\{\mathcal{G}^{n}(B)\right\}$ for all $B \in C L(M)$.

Proof Define the binary relation $\mathcal{R}$ on $C L(M)$ by

$B \mathcal{R} C \Leftrightarrow B \subset C$ for all $B, C \in C L(M)$.

Observe that $\mathcal{R}$ is transitive and hence $\mathcal{G}$-transitive. Now, we show that all the assumptions of Theorem 5.3 are fulfilled.

- In view of Theorem 3.2, for each $i \geq 1$, there is a unique fixed point $z_{i}$ of $f_{i}$. Therefore, for every nonempty $K \subset \mathbb{N},\left\{z_{i}: i \in K\right\}=\bigcup_{i \in K}\left\{f_{i}\left(z_{i}\right)\right\} \subset \bigcup_{i \geq 1} f_{i}\left(C_{K}\right)$, and hence $C_{K} \subset \mathcal{G}\left(C_{K}\right)$, so that assumption (a) is satisfied.

- for any $B, C \in C L(M), B \subset C$ clearly implies that $\mathcal{G}(B) \subset \mathcal{G}(C)$, and therefore assumption (b) holds.

- According to [30, 32], the completeness of $(M, d)$ implies the completeness of $(C L(M), \eta)$, and hence assumption (c) clearly holds.

- Let $B, C \in C L(M)$ be such that $C \subset B, \eta(B, C)<\infty$, and $\mathcal{G}(B) \neq \mathcal{G}(C)$. As $\mathcal{G}(B) \neq \mathcal{G}(C)$, we can find $i \in \mathbb{N}$ such that $f_{i}(B) \neq f_{i}(C)$, so that $D\left(f_{i}(B), f_{i}(C)\right)>0$. Hence, using Lemma 2.2, Lemma 6.1 and the continuity of $\theta$, we have

$$
\begin{aligned}
\theta(D(\mathcal{G}(B), \mathcal{G}(C))) & \leq \theta\left(\sup _{i \geq 1} D\left(f_{i}(B), f_{i}(C)\right)\right) \\
& \leq \sup _{i \geq 1} \theta\left(D\left(f_{i}(B), f_{i}(C)\right)\right) \\
& \leq \sup _{i \geq 1}[\theta(D(B, C))]^{h_{i}}=[\theta(D(B, C))]^{h},
\end{aligned}
$$

where in the last inequality we used (6.3). As $C \subset B$ and $\mathcal{G}(C) \subset \mathcal{G}(B)$, we have $D(C, B)=D(\mathcal{G}(C), \mathcal{G}(B))=0$, so that

$$
\theta(\eta(\mathcal{G}(B), \mathcal{G}(C))) \leq[\theta(\eta(B, C))]^{h}
$$

Therefore, assumption (d) is fulfilled. 
- Next, we show that assumption (e') is satisfied. To this end, let $\left\{B_{n}\right\}$ be a sequence of closed subsets of $M$ such that $B_{n} \subset B_{n+1}$ (for all $n \in \mathbb{N}$ ) and $\lim _{n \rightarrow \infty} B_{n}=B$ for some $B \in C L(M)$. We will show that $B_{n} \subset B$ for all $n \in \mathbb{N}$. Let $n_{0} \in \mathbb{N}$ and $b \in B_{n_{0}}$ be fixed. Observe that, for each $n \geq n_{0}$, we have $b \in B_{n}$ (as $B_{n_{0}} \subset B_{n}$ ). Therefore, for all $n \geq n_{0}$, we have

$$
\inf _{u \in B} d(b, u) \leq \sup _{v \in B_{n}} \inf _{u \in B} d(v, u)=D\left(B_{n}, B\right) \rightarrow 0 \quad \text { as } n \rightarrow \infty\left(\because \lim _{n \rightarrow \infty} B_{n}=B\right) .
$$

Hence $b \in \bar{B}=B$, and therefore $B_{n_{0}} \subset B$.

Now, conclusions (i) and (ii) follow from Theorem 5.2. Finally, if we assume that $\mathcal{G}(M)$ is bounded, then (i) does not occur. As $B \cup C \in C L(M)$ for all $B, C \in C L(M)$, the addition assumption of Theorem 5.3 is satisfied, and hence there exists a unique $A \in C L(M)$ such that $A=\overline{\bigcup_{i \geq 1} f_{i}(A)}$ and $\lim _{n \rightarrow \infty} \mathcal{G}^{n}(B)=A$ for all $B \in C L(M)$. This completes the proof.

In support of Theorem 6.1, we provide the following example.

Example 6.1 Let $M=[0, \infty)$ be equipped with the usual metric. For each $i \in \mathbb{N}$, define $f_{i}$ : $M \rightarrow M$ by $f_{i}(u)=\frac{u}{i u+1}+\beta_{i}$ for all $u \in M$, where $\beta_{i} \geq 0$. Consider the function $\theta:(0, \infty) \rightarrow$ $(1, \infty)$ given in Example 3.4. Then we have the following:

(i) for each $i \in \mathbb{N}, f_{i}$ is not a Banach contraction;

(ii) $\left\{f_{i}\right\}_{i \geq 1}$ is a weak $\theta$-CIFS, and for each $i \in \mathbb{N}, h_{i}=e^{-1} \in(0,1), h_{i}$ being the constant associated with $f_{i}$ from (3.1). Furthermore, if $C_{K}=\left\{\frac{i \beta_{i}+\sqrt{i^{2} \beta_{i}^{2}+4 i \beta_{i}}}{2 i} ; i \in K\right\}$ for every nonempty $K \subset \mathbb{N}$ and $\mathcal{G}: C L(M) \rightarrow C L(M)$ be defined as in Definition 6.1, then one of the following cases occurs:

(I) $\eta\left(\mathcal{G}^{n}\left(C_{K}\right), \mathcal{G}^{n+1}\left(C_{K}\right)\right)=\infty$ for all $n \in \mathbb{N}$; or

(II) there exists an attractor $A \in C L(M)$ of the considered weak $\theta$-CIFS and $A=\lim _{n \rightarrow \infty} \mathcal{G}^{n}\left(C_{K}\right)$, the limit being taken w.r.t. the generalized Hausdorff-Pompeiu metric.

(iii) if $\sup _{i \geq 1} \beta_{i}<\infty$, then the attractor $A$ is unique, and $A=\lim _{n \rightarrow \infty} \mathcal{G}^{n}(B)$ for all $B \in C L(M)$.

Proof In view of Example 3.7, for each $i \in \mathbb{N}, f_{i}$ is a weak $\theta$-contraction (with $h_{i}=e^{-1}$ ), not a Banach contraction, and its fixed point is $z_{i}=\frac{i \beta_{i}+\sqrt{i^{2} \beta_{i}^{2}+4 i \beta_{i}}}{2 i}$. Observe that $\sup _{i \geq 1} h_{i}=e^{-1}<1$ and, for each $i \in \mathbb{N}, f_{i}(M) \subset\left[\beta_{i}, \beta_{i}+\frac{1}{i}\right]$, so that $\mathcal{G}(M) \subset\left[0, \sup _{i \geq 1} \beta_{i}+1\right]$, and hence $\mathcal{G}(M)$ is bounded. Therefore, the conclusion of this example follows from Theorem 6.1.

Acknowledgements

All the authors are grateful to the anonymous referees for their excellent suggestions, which greatly improved the presentation of the paper.

Funding

This work was supported by Hajjah University (Yemen) and Integral University (India).

Competing interests

All the authors declare that they have no competing interests.

Authors' contributions

All the authors contributed equally and significantly in writing this article. All authors read and approved the final manuscript. 


\section{Author details}

'Department of Mathematics, Aligarh Muslim University, Aligarh, India. ${ }^{2}$ Department of Mathematics, Hajjah University, Hajjah, Yemen. ${ }^{3}$ Department of Mathematics, Integral University, Lucknow, India.

\section{Publisher's Note}

Springer Nature remains neutral with regard to jurisdictional claims in published maps and institutional affiliations.

\section{Received: 27 March 2018 Accepted: 21 November 2018 Published online: 28 November 2018}

\section{References}

1. Ran, A.C., Reurings, M.C.: A fixed point theorem in partially ordered sets and some applications to matrix equations. Proc. Am. Math. Soc., 132, 1435-1443 (2004)

2. Nieto, J.J., Rodríguez-López, R.: Contractive mapping theorems in partially ordered sets and applications to ordinary differential equations. Order 22(3), 223-239 (2005)

3. Wardowski, D.: Fixed points of a new type of contractive mappings in complete metric spaces. Fixed Point Theory Appl. 2012(94), 6 (2012)

4. Kirk, W., Srinivasan, P., Veeramani, P.: Fixed points for mappings satisfying cyclical contractive conditions. Fixed Point Theory 4(1), 79-89 (2003)

5. Jleli, M., Samet, B.: A new generalization of the Banach contraction principle. J. Inequal. Appl. 2014, 38 (2014)

6. Matkowski, J.: Fixed point theorems for mappings with a contractive iterate at a point. Proc. Am. Math. Soc. 62(2), 344-348 (1977)

7. Hutchinson, J.E.: Fractals and self similarity. Indiana Univ. Math. J. 30(5), 713-747 (1981)

8. Barnsley, M.F.: Fractals Everywhere. Revised with the Assistance of and with a Foreword by Hawley Rising, III. Academic Press Professional, Boston (1993)

9. Turinici, M.: Abstract comparison principles and multivariable Gronwall-Bellman inequalities. J. Math. Anal. Appl. 117(1), 100-127 (1986)

10. Nieto, J.J., Rodríguez-López, R.: Existence and uniqueness of fixed point in partially ordered sets and applications to ordinary differential equations. Acta Math. Sin. Engl. Ser. 23(12), 2205-2212 (2007)

11. Bhaskar, T.G., Lakshmikantham, V.: Fixed point theorems in partially ordered metric spaces and applications. Nonlinear Anal., Theory Methods Appl. 65(7), 1379-1393 (2006)

12. Ben-El-Mechaiekh, H.: The Ran-Reurings fixed point theorem without partial order: a simple proof. J. Fixed Point Theory Appl. 1(16), 373-383 (2014)

13. Samet, B., Vetro, C., Vetro, P.: Fixed point theorems for $\alpha-\psi$-contractive type mappings. Nonlinear Anal., Theory Methods Appl. 75(4), 2154-2165 (2012)

14. Alam, A., Imdad, M.: Relation-theoretic contraction principle. J. Fixed Point Theory Appl. 17(4), $693-702$ (2015)

15. Imdad, M., Khan, Q.H., Alfaqih, W.M., Gubran, R.: A relation theoretic (F, $\mathcal{R})$-contraction principle with applications to matrix equations. Bull. Math. Anal. Appl. 10(1), 1-12 (2018)

16. Imdad, M., Alfaqih, W.M.: A relation-theoretic expansion principle. Acta Univ. Apulensis 54, 55-69 (2018)

17. Gubran, R., Imdad, M., Khan, I.A., Alfaqih, W.M.: Order-theoretic common fixed point results for F-contractions. Bull. Math. Anal. Appl. 10(1), 80-88 (2018)

18. Rus, I.A.: Picard operators and applications. Sci. Math. Jpn. 58(1), 191-219 (2003)

19. Berzig, M., Karapınar, E., Roldán-López-de-Hierro, A.-F.: Discussion on generalized- $(\alpha \psi, \beta)$-contractive mappings via generalized altering distance function and related fixed point theorems. In: Abstract and Applied Analysis, vol. 2014 Hindawi Publishing Corporation, Egypt (2014)

20. Secelean, N.A.: Countable Iterated Function Systems. LAP LAMBERT Academic Publishing, (2013)

21. Secelean, N.A.: Iterated function systems consisting of F-contractions. Fixed Point Theory Appl. 2013, 277 (2013)

22. Diaz, J., Margolis, B.: A fixed point theorem of the alternative, for contractions on a generalized complete metric space. Bull. Am. Math. Soc. 74(2), 305-309 (1968)

23. Piri, H., Kumam, P.: Some fixed point theorems concerning F-contraction in complete metric spaces. Fixed Point Theory Appl. 2014, 210 (2014)

24. Hussain, N., Parvaneh, V., Samet, B., Vetro, C.: Some fixed point theorems for generalized contractive mappings in complete metric spaces. Fixed Point Theory Appl. 2015, 185 (2015)

25. Li, Z., Jiang, S.: Fixed point theorems of JS-quasi-contractions. Fixed Point Theory Appl. 2016(1), 40 (2016)

26. Parvaneh, V., Golkarmanesh, F., Hussain, N., Salimi, P.: New fixed point theorems for $(\alpha-\Theta)$-contractions in ordered metric spaces. J. Fixed Point Theory Appl. 18(4), 905-925 (2016)

27. Zhenga, D., Wangb, P.: Weak $\theta-\phi$-contraction and discontinuity. J. Nonlinear Sci. Appl. 10(5), 2318-2323 (2017)

28. Altun, I., Al Arifi, N., Jleli, M., Lashin, A., Samet, B.: A fixed point theorem for JS-contraction type mappings with applications to polynomial approximations. Filomat 31, 15 (2017)

29. Hussaina, N., Al-Mazrooeib, A.E., Ahmadb, J.: Fixed point results for generalized $(\alpha-\eta)-\theta$ contractions with applications. J. Nonlinear Sci. Appl. 10(8), 4197-4208 (2017)

30. Secelean, N.-A.: Weak F-contractions and some fixed point results. Bull. Iran. Math. Soc. 42(3), 779-798 (2016)

31. Gordji, M.E., Ramezani, M., Sajadian, F., Cho, Y.J., Park, C.: A new type fixed point theorem for a contraction on partially ordered generalized complete metric spaces with applications. Fixed Point Theory Appl. 2014(1), 15 (2014)

32. Beer, G.: Topologies on Closed and Closed Convex Sets, Vol. 268 Springer Science \& Business Media (1993)

33. Secelean, N.-A., Wardowski, D.: \&F-contractions: not necessarily nonexpansive Picard operator. Results Math. 70(3-4), 415-431 (2016)

34. Radenovic, S., Vetro, F., Vujaković, J.: An alternative and easy approach to fixed point results via simulation functions. Demonstr. Math. 50(1), 223-230 (2017)

35. Kadelburg, Z., Radenovic, S., Shukla, S.: Boyd-Wong and Meir-Keeler type theorems in generalized metric spaces. J. Adv. Math. Stud. 9(1), 83-93 (2016)

36. Došenovic, T., Radenovic, S.: A comment on "Fixed point theorems of JS-quasi-contractions". Indian Math. Society (2018, to appear) 